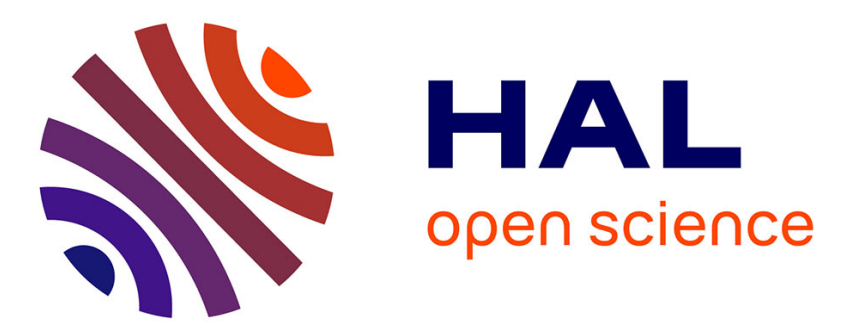

\title{
Analyse régionale sur les extrêmes hydrométriques en France: détection de changements cohérents et recherche de causalité hydrologique
}

\author{
M. Lang, Benjamin Renard
}

\section{- To cite this version:}

M. Lang, Benjamin Renard. Analyse régionale sur les extrêmes hydrométriques en France: détection de changements cohérents et recherche de causalité hydrologique. La Houille Blanche - Revue internationale de l'eau, 2007, 6, p. 83 - p. 89. 10.1051/lhb:2007087 . hal-00453855

\section{HAL Id: hal-00453855 \\ https://hal.science/hal-00453855}

Submitted on 5 Feb 2010

HAL is a multi-disciplinary open access archive for the deposit and dissemination of scientific research documents, whether they are published or not. The documents may come from teaching and research institutions in France or abroad, or from public or private research centers.
L'archive ouverte pluridisciplinaire HAL, est destinée au dépôt et à la diffusion de documents scientifiques de niveau recherche, publiés ou non, émanant des établissements d'enseignement et de recherche français ou étrangers, des laboratoires publics ou privés. 


\title{
Analyse régionale sur les extrêmes hydrométriques en France : détection de changements cohérents et recherche de causalité hydrologique
}

\author{
Regional analysis on changes in hydrological extremes in France \\ MICHEL LANG, BENJAMIN RENARD \\ CEMAGREF Lyon, 3 bis quai Chauveau, 69009 Lyon \\ Tél : +33 (0)4 722087 98, Fax : +33 (0)4 784778 75, e-mail : lang@lyon.cemagref.fr
}

\begin{abstract}
$T$ This paper presents a national study on the detection of climatic changes in hydrological extremes in France. A high number of changes have been detected but after an extensive criticism of data, half of these changes can be related to non-climatic factors, especially metrological issues. A regional analysis based on a reduced number of stations, whose quality can be insured, doesn't show a consistent and generalized change in French rivers. Specific behaviours have been explained in mountainous basins, related to the increasing of temperature, and in the North of France with an increasing of both rainfall and flood peaks.
\end{abstract}

\section{I INTRODUCTION}

Le changement climatique est une réalité largement reconnue aujourd'hui dans la communauté scientifique. Néanmoins, son impact sur les extrêmes hydrométriques est délicat à observer. La première difficulté vient de la forte variabilité inter-annuelle naturelle du régime des rivières, qui peut masquer une évolution du second ordre sur les valeurs extrêmes. Il est dès lors indispensable d'exploiter de longues séries de données pour pouvoir espérer détecter une évolution dans les séries. La forte variabilité spatiale de la climatologie et des propriétés physiographiques des bassins versants et un comportement fortement non linéaire induisent une réponse très variable sur les changements à attendre sur le régime des rivières.

Un premier travail a été réalisé de 2002 à 2004 dans le cadre d'un projet de recherche financé par le PNRH, pour la mise au point de techniques de détection de changements dans les séries chronologiques et un premier diagnostic sur les effets observés du changement climatique sur les crues et les étiages. Il a ensuite été approfondi et généralisé à l'ensemble de la France métropolitaine, au cours du travail de thèse de $\mathrm{B}$. Renard (2006). Les premiers résultats de ces travaux ont été présentés par Renard et al. [7] lors d'un précédent colloque de la Société Hydrotechnique de France, avec des informations sur la procédure de détection de changements, une analyse locale sur l'évolution du régime des extrêmes en France, et une approche Bayésienne sur un calcul probabiliste des valeurs extrêmes en contexte non stationnaire. Après un rappel rapide sur ces travaux, nous présentons les nouveaux résultats issus d'une analyse régionale de stationnarité, en s'intéressant à la significativité des résultats à l'échelle de la France, à la cohérence régionale des évolutions détectées, et nous terminons par la recherche de causalité hydrologique sur une région hydroclimatique.

\section{II $\square$ RAPPEL SUR LES RÉSULTATS DE LANALYSE LOCALE DE STATIONNARITÉ}

\section{II.1 PREMIÈRE ANALYSE SUR UN JEU DE 200 LONGUES SÉRIES HYDROMÉTRIQUES}

Un jeu de 192 longues séries de débits journaliers a été constitué sur la France métropolitaine, en retenant les séries réputées non influencées, disposant d'au moins 40 années d'observations et dont les données sont jugées de bonne qualité par les gestionnaires hydrométriques. Une trentaine d'échantillons a alors été extrait pour chacune des stations sélectionnées, à partir de variables descriptives des hauteseaux (pointe et hydrogramme de crue, date et fréquence d'apparition), des basses-eaux (débit minimal sur 1 et 7 jours, date d'étiage) et du régime des eaux (module et variabilité interannuelle). Chacun de ces échantillons a alors fait l'objet d'un test de stationnarité choisi en fonction de l'autocorrélation de la série, du type de distribution suivie par la variable étudiée (loi de Gauss ou loi des valeurs extrêmes, loi inconnue), du type de changement attendu (rupture, tendance ou non connu) et de la longueur de l'échantillon. Pour chaque station, les résultats ont été regroupés dans une «fiche-station », où des vignettes représentent la chronologie de la variable étudiée, ainsi que la tendance ou la rupture estimée, avec la significativité du test (1,5 ou $10 \%)$, ainsi que la date de rupture estimée. La synthèse des résultats au niveau français (cf. Figure 1) montre qu'en crue environ un quart des 179 stations traitées présente des séries significativement non stationnaires, au risque d'erreur de $10 \%$, alors que la proportion passe à un tiers des 128 stations pour les variables d'étiages. On ne relève pas de cohérence dans la répartition spatiale des anomalies détectées, ni de changement généralisé à la hausse pour les crues. Les changements les plus nombreux concernent les basses-eaux, en particulier sur la valeur minimale du débit moyen d'étiage sur 7 ou 30 jours. 

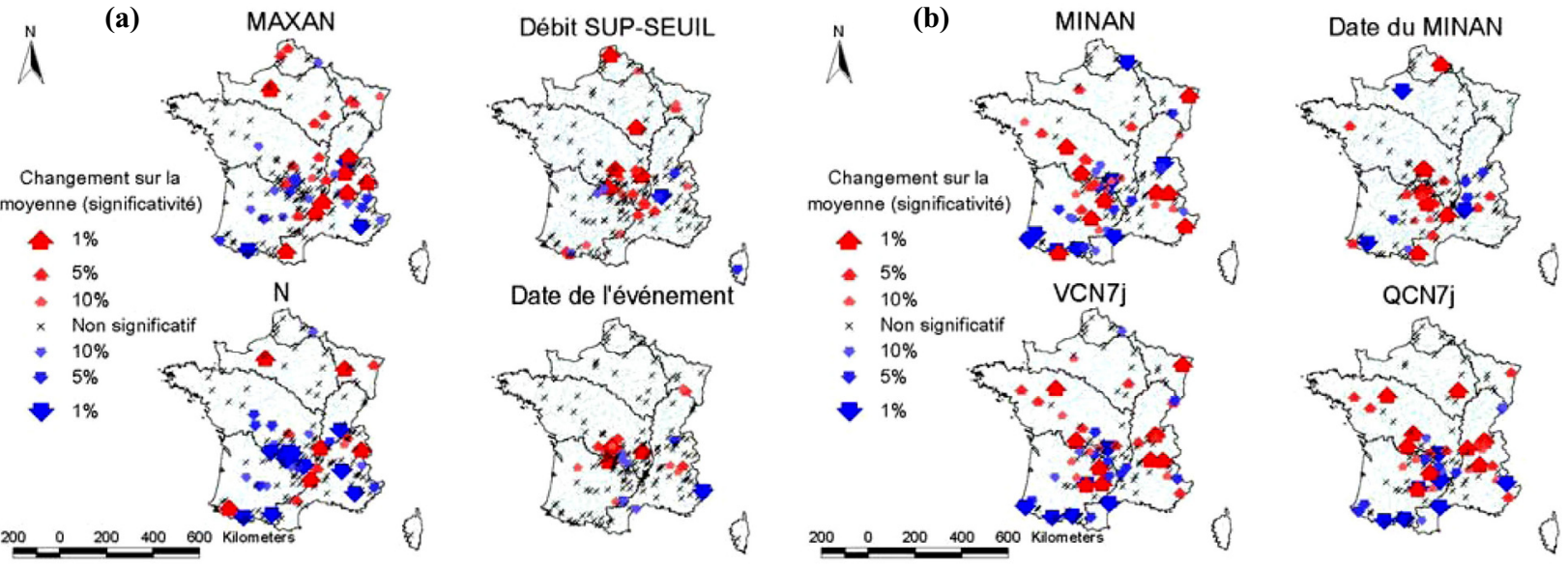

Figure 1 : Résultat des tests de stationnarité sur le premier jeu de données.

(a) Test sur quatre variables de crue : MAXAN (maximum annuel des débits journaliers), débit SUP-SEUIL (pointe de débit journalier supérieure à un seuil), $\mathbf{N}$ (nombre annuel d'événements supérieurs au seuil), Date des événements supérieurs au seuil. Le seuil est choisi de façon à obtenir un événement par an en moyenne.

(b) Test sur quatre variables de basses-eaux : MINAN (minimum annuel des débits journaliers), date du MINAN, VCN7j (minima annuel des débits moyens sur 7 jours) et QCN7j (minima annuel des débits seuils sur 7 jours).

\section{II.2 RETOUR AUPRÈS DES GESTIONNAIRES HYDROMÉTRIQUES ET SECONDE ANALYSE}

L'ensemble des résultats précédents a été publié sur Internet : http ://www.lyon.cemagref.fr/hh/PNRH-NS, et une visite ou un contact téléphonique ont été programmés auprès de chaque gestionnaire hydrométrique pour interpréter l'origine des changements détectés sur les séries. L'objectif était d'éliminer les stations pour lesquelles une explication d'origine anthropique peut être avancée. Près de la moitié des ruptures observées sont en fait imputables à un changement dans la chaîne de traitement hydrométrique (notamment l'extrapolation de la courbe de tarage en crue) ou à la présence d'aménagements modifiant les basses-eaux et non identifiés dans le premier jeu de données. A l'issue de cette étape de critique des données, le nombre de séries finalement utilisa- bles est de 149 pour les hautes-eaux (dont 25 pour les crues nivales), 90 pour les basses-eaux et 130 pour la description des régimes. La réactualisation des cartes de synthèse (cf. Figure 2), avec un nombre bien plus réduit de changements significatifs, ne présente pas de cohérence spatiale.

\section{III $\square$ ANALYSE REGIONALE DE STATIONNARITÉ EN FRANCE MÉTROPOLITAINE}

\section{III.1 SIGNIFICATIVITÉ À L'ÉCHELLE DE LA FRANCE}

Nous allons apprécier si le nombre de changements qui vient d'être détecté localement avec un risque d'erreur $\alpha$ est
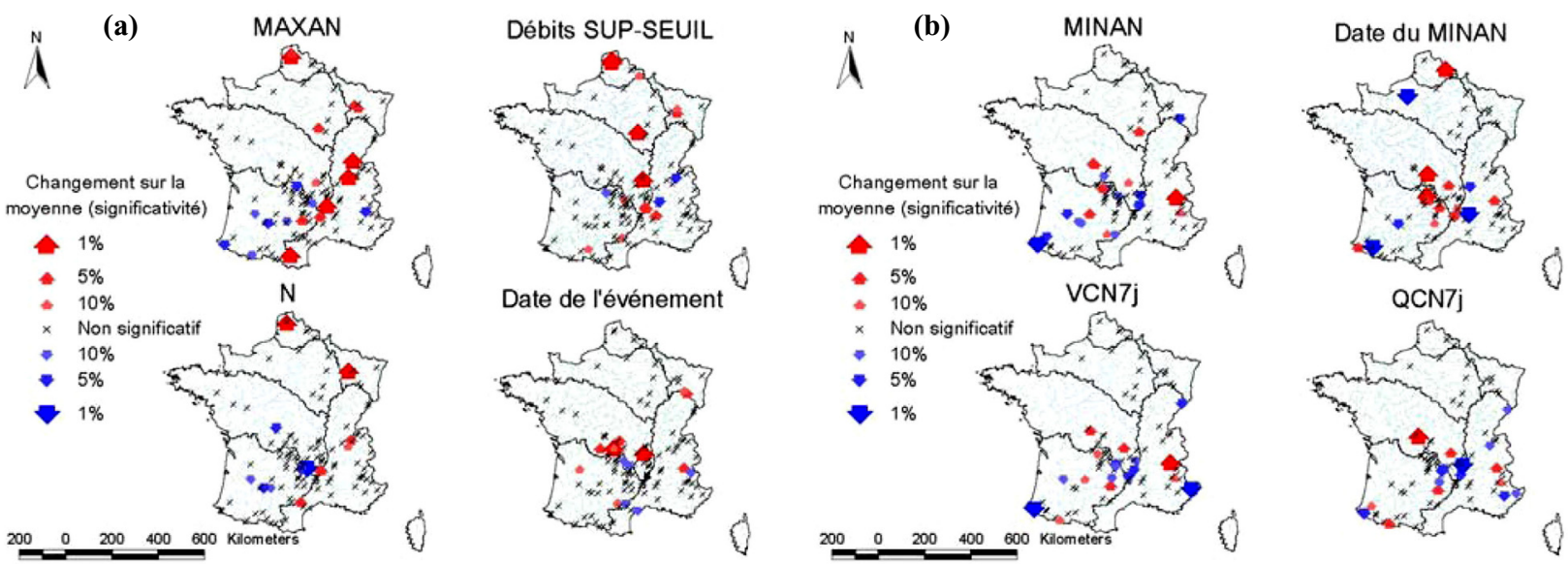

Figure 2 : Résultat des tests de stationnarité sur le second jeu de données (après critique des données). (a) Test sur quatre variables de crue. (b) Test sur quatre variables de basses-eaux. 
significatif à un niveau national. Supposons que l'on traite un jeu de 100 stations stationnaires avec un risque d'erreur $\alpha=0.05$ : on s'attend à détecter à tort un changement sur 5 stations. A partir de combien de résultats significatifs $N$ peut-on affirmer, avec un risque d'erreur $\alpha$ 'que tous ces changements ne peuvent être dus au hasard ? Dans le cas où les données de toutes les stations sont supposées indépendantes, on peut facilement montrer que le nombre $N$ suit une loi binomiale : $\operatorname{Pr}(N=k)=C_{p}^{k} \alpha^{k}(1-\alpha)^{p-k}$, avec pour l'exemple de 100 tests avec un risque $\alpha=0.05$ un nombre minimal $N_{\alpha}=9$ pour un risque $\alpha^{\prime}=0.05: \operatorname{Pr}(N \leq 8)=0.937$ et $\operatorname{Pr}(N \leq 9)=0.972$. Dans la pratique, il est nécessaire de tenir compte du certain niveau d'auto-corrélation présent dans les séries testées. Une approche simplifiée consiste à raisonner en terme d'effectif efficace, en considérant que $p$ stations auto-corrélées sont équivalentes à $p^{*}$ stations indépendantes, avec $p / p^{*}=1+(p-1) \rho$, où $\rho$ est la moyenne des coefficients de corrélation entre chaque couple de stations (Douglas et al., 2000 ; Yue et Wang, 2002). Pour une corrélation moyenne $\rho=0.1$, le nombre équivalent de stations indépendantes vaut $p^{*}=9.17$. La valeur critique de 2.37 stations indépendantes correspond à un effectif critique de $2.37 / 9.17=25.8 \%$. Le nombre minimal $N_{\alpha}$, passe ainsi de 9 à 26 détections.

Des simulations effectuées par Renard (2006) ont montré que cette approche simplifiée ne prend qu'imparfaitement en compte l'effet de la dépendance spatiale. Une approche plus complète consiste à : (a) simuler une série de jeux régionaux de données stationnaires conservant la même structure de dépendance spatiale que les observations $\left(m=1, N_{\text {sim }}\right.$ simulations); (b) appliquer pour chaque jeu simulé $m$ un test à chacune des $p$ stations, et compter le nombre $N(m)$ de changements significatifs détectés; (c) calculer le nombre critique $N_{\alpha}$, à partir de la distribution des valeurs $N(m)$ et le comparer avec le nombre de changements observés. La procédure de simulations peut être basée sur une copule Gaussienne faisant une hypothèse sur la structure de dépendance spatiale (Renard et Lang, 2007), ou sur une procédure de réchantillonnage par Bootstrap-année (Douglas et al., 2000). Le Tableau 1 résume les résultats obtenus sur le second jeu de données, par Bootstrap sur la période commune d'observation 1965-2000. Les tests ont été effectués localement avec un risque $\alpha=0.05$ et interprétés nationalement avec un risque $\alpha^{\prime}=0.05$. On constate que le nombre de changements détectés localement n'est pas significatif nationalement. En d'autres termes, il n'y a pas suffisamment de changements détectés au risque d'erreur de $5 \%$ sur la Figure 2 pour garantir qu'ils ne sont pas tous dus au hasard.

\section{III.2 COHÉRENCE RÉGIONALE}

En l'absence d'évolution généralisée constatée sur les débits, nous nous intéressons maintenant à l'analyse de la cohérence régionale des évolutions détectées. Une série de régions hydro-climatiques a été établie par Renard (2006) en croisant une classification basée sur la saisonnalité des crues et des étiages avec un zonage pluviométrique de MétéoFrance (Champeaux et Tamburini, 1995). La Figure 3 illustre la localisation des stations étudiées suivant la région hydro-climatique. Quand cela était possible, l'analyse de la cohérence régionale a été réalisée sur des variables définies régionalement, comme par exemple la date moyenne d'occurrence du maximum annuel, retenue comme indicatrice de la saisonnalité des crues sur une région. Dans le cas contraire où l'agglomération des données à l'échelle d'une région n'est pas aisée (cf. le cas du débit de pointe, fortement lié à la superficie du bassin versant), une alternative consiste à définir une statistique régionale à partir de la moyenne des statistiques locales. Douglas et al. (2000) et Yue et Wang (2002) ont ainsi proposé une version régionale du test de Mann et Kendall. Il subsiste toutefois une difficulté analogue à celle mentionnée pour la significativité régionale, à savoir la prise en compte de la dépendance spatiale entre sites. Un exemple de ce problème peut être donné en considérant dix stations fortement liées, affectées d'une tendance à la hausse, et une onzième affectée d'une tendance à la baisse. Les deux approches précédentes seront biaisées dans la mesure où l'information des dix stations corrélées va masquer l'information contenue dans le onzième échantillon.

Renard (2006) a proposé un test régional de déviance, pour comparer deux modèles emboîtés $M_{0}$ et $M_{1}$, le premier modèle stationnaire étant un sous-modèle du second. L'approche consiste à transformer par anamorphose la matrice $X$ ( $n$ années en ligne et $p$ stations en colonne) en matrice $\tilde{\mathbf{X}}$

Tableau 1. Significativité à l'échelle de la France des changements observés. Risque local et national égal à $5 \%$.

\begin{tabular}{|c|c|c|c|c|c|}
\hline & $\begin{array}{c}\text { Nombre } \\
\text { de stations }\end{array}$ & $\begin{array}{c}\text { Nombre d'années } \\
\text { communes }\end{array}$ & Variable testée & $\%$ critique $N_{0.05}$ & \% observé \\
\hline \multirow{2}{*}{ Crues } & \multirow{2}{*}{83} & \multirow{2}{*}{36} & $M A X A N$ & 19.3 & 12.1 \\
\hline & & & Date du MAXAN & 18.1 & 3.2 \\
\hline \multirow[t]{2}{*}{ Crues nivales } & \multirow[t]{2}{*}{15} & \multirow[t]{2}{*}{41} & $\begin{array}{c}\text { Débit seuil } \\
Q C X 10 j\end{array}$ & 33.3 & 8.0 \\
\hline & & & Date du $Q C X 10 j$ & 33.3 & 28.0 \\
\hline \multirow{2}{*}{ Etiages } & \multirow{2}{*}{58} & \multirow{2}{*}{36} & MINAN & 22.4 & 12.2 \\
\hline & & & Date du MINAN & 22.4 & 6.7 \\
\hline Régime & 86 & 36 & Module & 26.7 & 2.3 \\
\hline
\end{tabular}



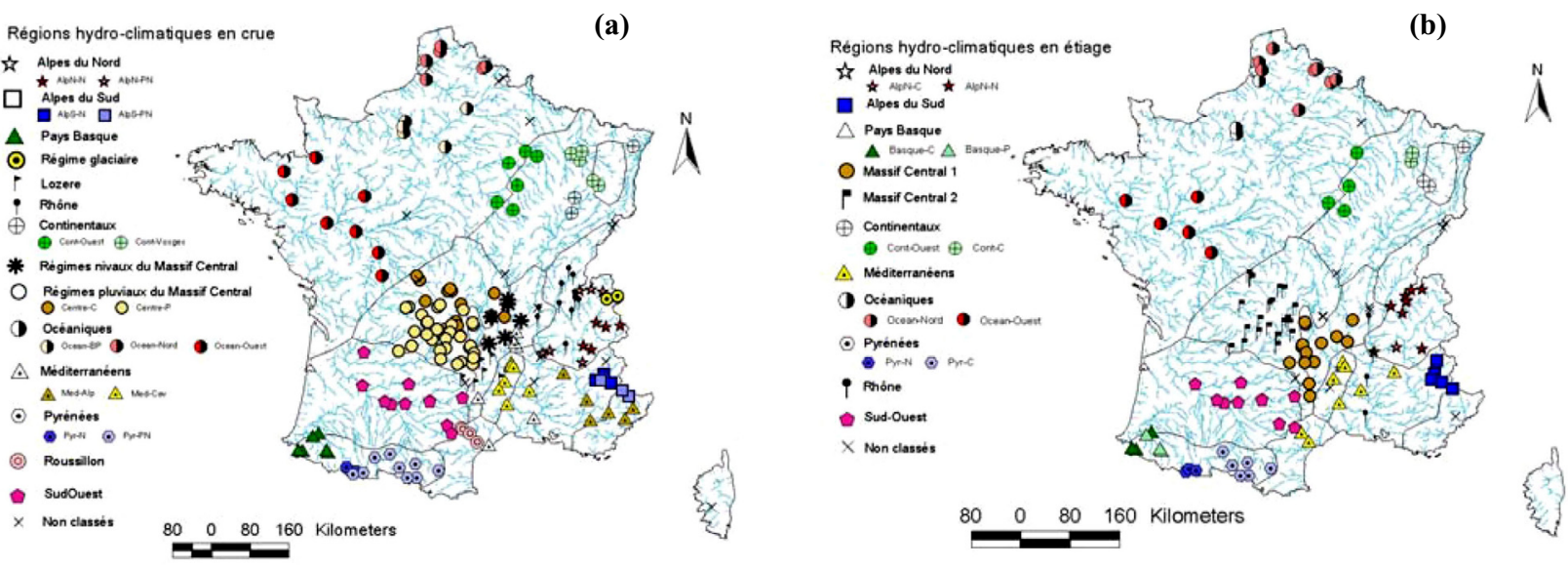

Figure 3 : Localisation des stations étudiées suivant leur région hydro-climatique. (a) classification pour les crues. (b) classification pour les étiages.

de variables centrées réduites de Gauss. On suppose que l'on peut alors modéliser la loi multivariée des valeurs de $\tilde{\mathbf{X}}$ par une loi normale de dimension $p: N(\mu ; \Sigma)$. Sous hypothèse de stationnarité (modèle $M_{0}$ ), le vecteur moyenne $\mu$ est nul, et la matrice de variance $\Sigma$ est obtenue par l'estimation du maximum de vraisemblance : $\boldsymbol{\Sigma}=(1 / n) \tilde{\mathbf{X}}^{t} \tilde{\mathbf{X}}$. Le modèle $M_{1}$ testé consiste à supposer que toutes les données sont affectées d'une tendance identique $\alpha: E\left(\tilde{X}_{k}^{(i)}\right)=\alpha \tilde{y}_{k}$, où $\tilde{X}_{k}^{(i)}$ est la variable transformée du site $i$ pour l'année $k$, et $\tilde{y}_{k}=y_{k}-\bar{y}$, où $y_{k}$ représente l'année associée à la $k^{e}$ donnée. Un estimateur $\hat{\alpha}$ de la tendance régionale est donné par Renard (2006). On peut alors calculer la fonction de déviance $T$, qui suit asymptotiquement une loi du Khi-deux à un degré de liberté :

$T=-2\left(L_{0}\left(\mathbf{X}, \hat{\boldsymbol{\theta}}_{0}\right)-L_{1}\left(\mathbf{X}, \hat{\boldsymbol{\theta}}_{1}\right)\right)=$

$$
-2\left(\sum_{k=1}^{n} \log N\left(\tilde{\mathbf{X}}_{k} ; 0, \hat{\boldsymbol{\Sigma}}\right)-\sum_{k=1}^{n} \log N\left(\tilde{\mathbf{X}}_{k} ; \alpha \tilde{y}_{k}, \hat{\boldsymbol{\Sigma}}\right)\right)
$$

L'hypothèse de normalité multivariée de la matrice $\tilde{\mathbf{X}}$ a été vérifiée sur l'ensemble des 15 régions hydro-climatiques et s'est révélée généralement satisfaisante. Dans le cas contraire, la cohérence régionale a été étudiée à l'aide d'une variable régionale ou à partir de la version régionale du test de Mann et Kendall. Nous avons pu détecter des évolutions sur seulement cinq des quinze régions hydro-climatiques, avec : (a) des étiages moins sévères dans les Alpes. De plus, dans les Alpes du Nord, l'onde de fonte nivale semble plus précoce; (b) une augmentation du module nival des stations glaciaires, accompagnée d'une précocité accrue de la date du maximum du débit de base; (c) une aggravation des étiages dans les Pyrénées et le Pays Basque. Les crues pluviales semblent également en diminution dans les Pyrénées ; (d) une augmentation des maxima annuels dans la région continentale du Nord-Est; (e) une aggravation des crues en région océanique (Nord de la France), apparemment liée au remplissage des nappes d'accompagnement.

A titre d'illustration, nous présentons les résultats sur deux de ces régions, le détail figurant dans la thèse de Renard (2006). Sur les Alpes du Nord (Figure 4a), les changements concernent les crues nivales et les étiages. L'onde de fonte est plus précoce, et le pic de fonte diminue. Les étiages sont moins sévères, avec une augmentation significative des minima annuels journaliers et une diminution significative du déficit de volume. La durée des étiages semble également décroître, mais pas de manière significative. Sur la zone continentale, les résultats obtenus sur la région complète sont les mêmes que ceux présentés ici (Figure 4b) sur les sous-régions « Ouest» et «Vosges » : bien que la significativité des évolutions diffère suivant l'échelle considérée, tous les changements vont dans le même sens et indiquent une augmentation de l'intensité des crues.

\section{IV — RECHERCHE DE CAUSALITES HYDROLOGIQUES}

L'étude régionale a permis de mettre en avant quelques régions présentant des changements cohérents. Ceci incite à penser que ces changements sont bien réels, et ne sont pas dus à des problèmes métrologiques ou des influences directes liées à des aménagements, qui ne devraient pas a priori exercer une influence régionale sur les débits. Cependant, une étude uniquement basée sur des données hydrométriques ne suffit pas à affirmer que ces évolutions sont climatiques. En effet, le rôle filtre joué par le bassin versant est une composante importante dans la transformation pluie-débit. Or, en France, rares sont les bassins versants n'ayant pas évolué au cours du vingtième siècle (urbanisation, agriculture, déforestation ou reboisement, etc.). L'impact de ces changements affectant le bassin versant est difficilement quantifiable, en particulier pour les événements extrêmes, mais ne peut être exclu. Il apparaît donc nécessaire d'utiliser des données climatologiques afin de faire la part de ce qui relève de la variabilité climatique (température, précipitation) de ce qui relève 
(a)
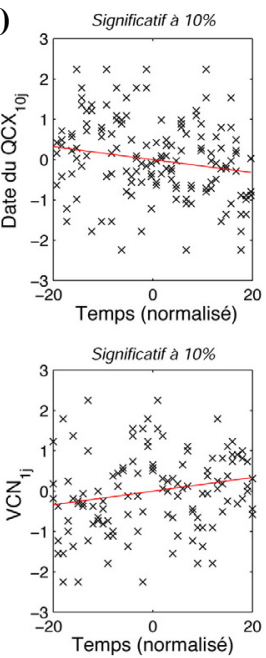

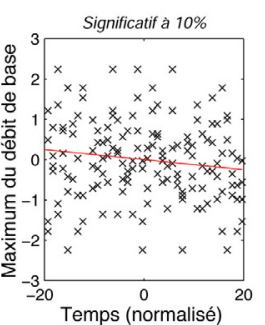

Significatif à $5 \%$

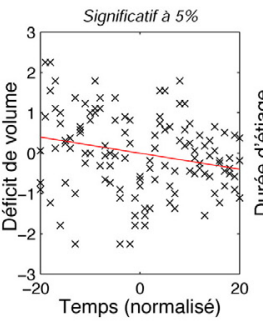

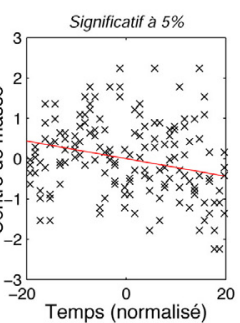

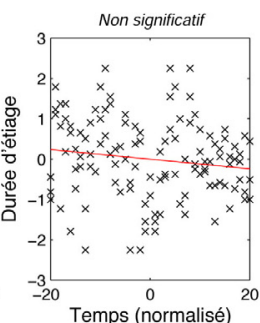

(b)
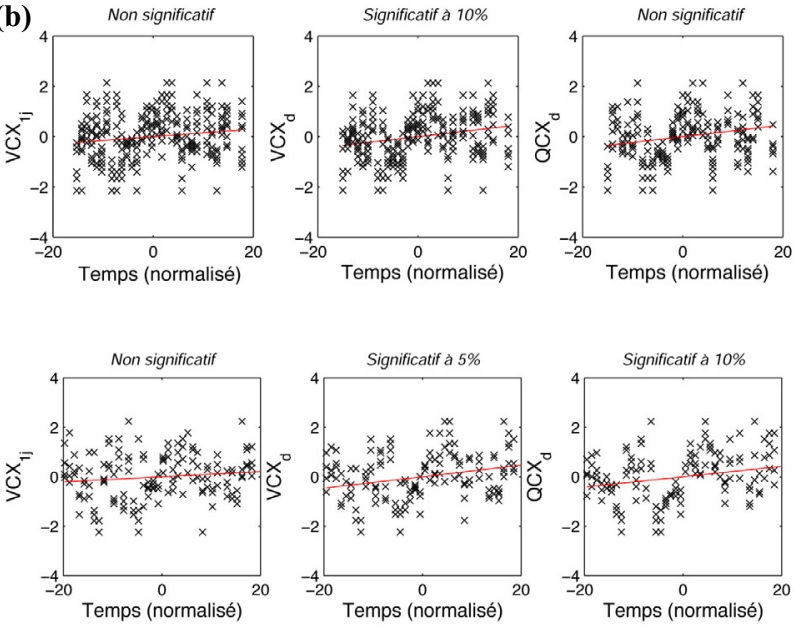

Figure 4 : Evolution régionale sur deux régions hydro-climatiques. Les variables sont transformées par normal score, et le temps centré sur zéro. (a) Alpes du Nord (QCX10j : débit seuil de crue dépassé 10 jours consécutifs ; VCN1j : débit journalier d'étiage). (b) Sous-régions « Ouest» "Vosges» de la zone continentale (VCXd et QCXd : débit moyen et débit seuil maximal calculés sur la durée moyenne d de dépassement de la moitié des pics de crue).

de changements dans l'occupation des sols. Une comparaison empirique avec les cartes d'évolution climatique mises au point dans le cadre du projet IMFREX (http ://medias.dsi. cnrs.fr/imfrex/web/index) ne donne pas de relation simple. Si le réchauffement constaté est significatif sur un grand nombre de postes et sur plusieurs indices (notamment le nombre de jours chaud supérieurs à $25^{\circ} \mathrm{C}$ ou le nombre de jours de gel), l'évolution des précipitations est bien moins marquée et n'est significative que pour des cumuls pluviométriques sur plusieurs journées. Ces éléments sont insuffisants pour expliquer des évolutions sur les extrêmes hydrométriques, en raison de la forte non-linéarité de la relation pluie-température-débit, et aussi à cause d'un manque de couverture spatiale pour les résultats relatifs aux variables extrêmes.

Une analyse plus détaillée est possible en comparant l'évolution observée des débits avec celle obtenue par une modélisation hydrologique. Deux exemples d'application ont été réalisés par Renard (2006), le premier sur l'évolution des crues pluviales dans la région continentale à partir du modèle GR4J (Perrin, 2000), le second sur l'évolution des crues nivales dans les Alpes à partir du modèle MORDOR
(Paquet, 2004). Nous présentons ici les résultats obtenus sur quatre bassins versants de la région continentale (bassin de la Moselle), à partir de données hydrométriques, pluviométriques et d'évapotranspiration potentielle sur la période 1968-1998. Un test régional de déviance sur les précipitations ne montre pas de tendance significative à la hausse au seuil de $10 \%$, sauf pour le nombre annuel de jours de fortes pluies, avec plus de $10 \mathrm{~mm}\left(R_{10 \mathrm{~mm}}\right)$ (cf. Figure 5). Les paramètres du modèle GR $4 \mathrm{j}$ ont ensuite été calés sur trois sous-périodes distinctes : 1968-1979, 1978-1989, 1988-1998, la première année de chaque période étant dédiée à l'initialisation de l'état de saturation du modèle.

La Figure 6 présente le résultat des tests de stationnarité qui montre une tendance significative et cohérente à la hausse, sur les observations (a) et sur deux sous-périodes (b et d), la hausse sur la seconde sous-période (c) n'étant pas significative. Ceci nous laisse à penser que l'évolution observée sur les débits est liée, au moins en partie, à une évolution affectant les précipitations. Afin de déterminer si l'aggravation observée sur le maximum annuel des débits journaliers résulte seulement d'une augmentation des pré-
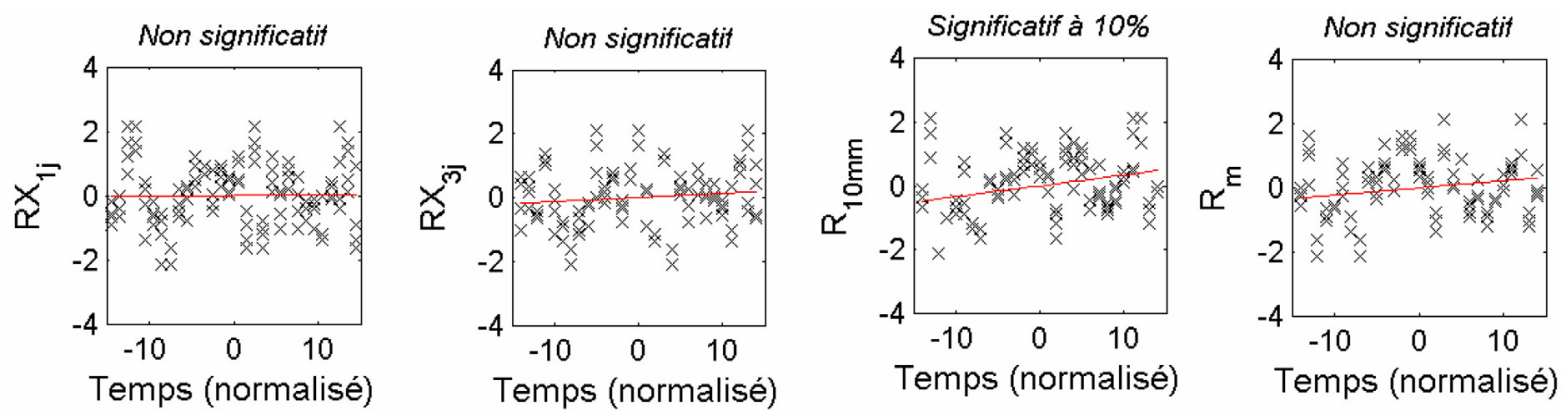

Figure 5 : Evolution régionale des précipitations sur le bassin de la Moselle : RX1j et RX3j, maxima annuel des pluies de 1 et 3 jours ; R10mm, nombre de jours avec plus de $10 \mathrm{~mm}$, R, pluie annuelle. 

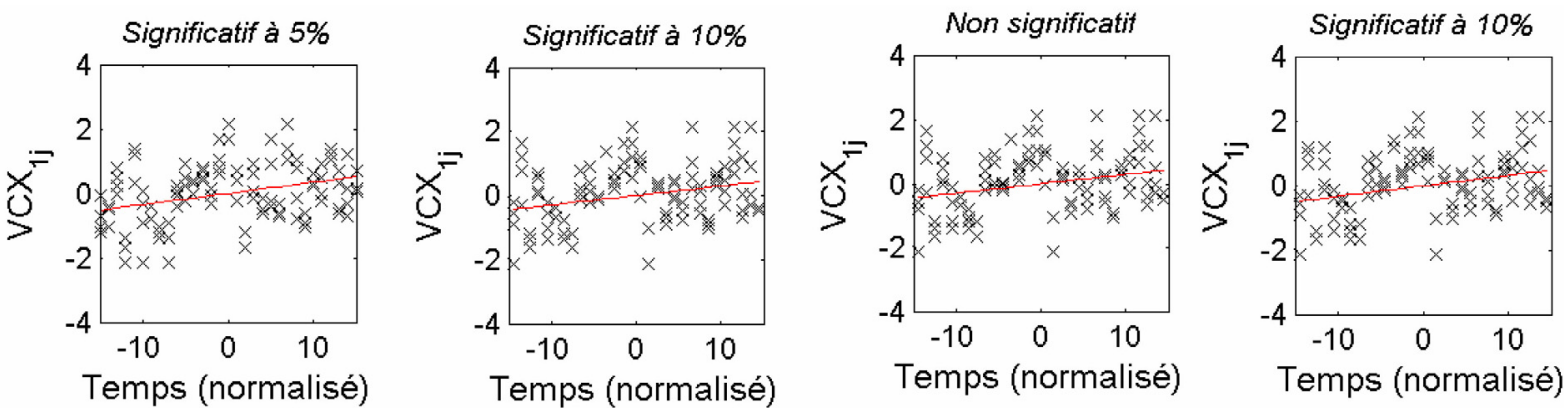

Figure 6 : Evolution régionale du maximum annuel des débits journaliers sur 4 bassins de la Moselle : (a) observations, (b-c-d) simulation GR4J avec calage respectivement sur 1968-1979, 1978-1989, 1988-1998.

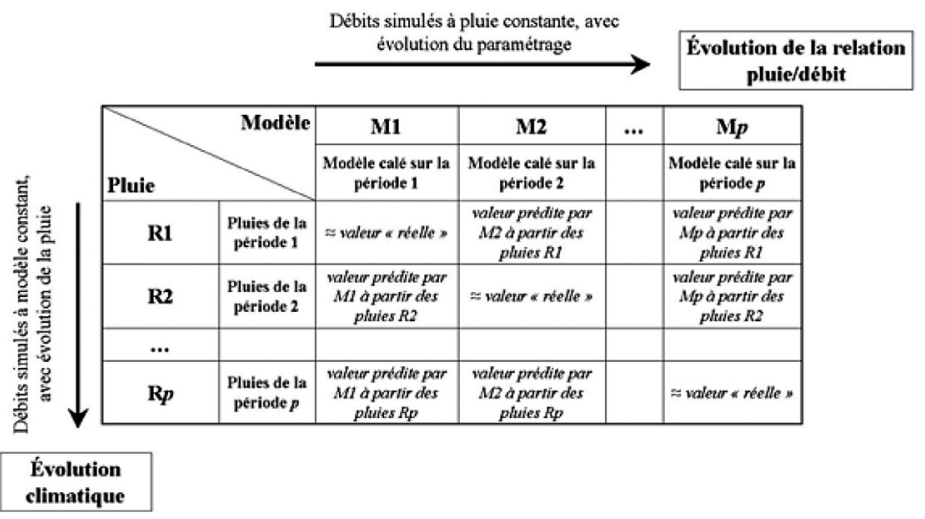

$S=\sum_{i=1}^{p}\left[\sum_{j=1}^{i-1}\left(q_{i i}-q_{i j}\right)+\sum_{j=i+1}^{p}\left(q_{i j}-q_{i i}\right)\right]$

$q_{i j}$ correspond à l'élément de la ligne $i$ et de la colonne $j$ de la matrice

Figure 7 : Principe des simulations croisées (d'après Andréassian et al., 2001) et statistique $S$ utilisée.

cipitations ou aussi d'un changement sur le processus de génération des débits, nous allons utiliser un test proposé par Andréassian et al. (2003) basé sur la modélisation hydrologique. Le principe de cette méthode repose sur des simulations croisées (Figure 7). La période d'étude est découpée en $p$ sous-périodes. Sur chacune de ces sous-périodes, les paramètres du modèle sont calés, et des débits sont simulés à partir des pluies $R 1, \ldots, R p$ observées. On obtient donc une matrice dont l'élément figurant en ligne $i$ et en colonne $j$ est lié aux débits simulés à partir des pluies de la période $i$ par le modèle calé sur la période $j$. Si l'on considère cette matrice par colonne, les débits simulés sont relatifs à un calage donné, et à l'ensemble des pluies observées. Les éventuelles évolutions seront donc d'origine pluviométrique. C'est cette évaluation qui a été réalisée dans la section précédente, avec trois sous-périodes de calage. Si la matrice est considérée par ligne, alors les débits simulés seront relatifs à une pluie donnée, pour différents jeux de paramètres calés. Une éventuelle évolution sera donc liée à une modification des paramètres du modèle, et donc de la relation pluie-débit.

La variable testée est ici le volume total écoulé au-dessus d'un seuil de haut débit (quantile $95 \%$ de la courbe des débits classés), avec six sous-périodes de cinq années. Une valeur critique de la statistique peut alors être estimée en recréant un grand nombre de matrices par permutation des colonnes de la matrice originale. Sous l'hypothèse $H_{0}$ d'absence d'évolution dans la relation pluie-débit, la valeur observée de la statistique devrait être comprise entre les quantiles de probabilité $\alpha / 2$ et $1-\alpha / 2$ de la distribution des statistiques obtenues par permutation ( $\alpha$ étant le risque de première espèce). Les résultats illustrés sur la Figure 8 montrent que l'hypothèse de stationnarité de la relation pluie-débit ne peut être rejetée sur trois des quatre stations testées. L'utilisation du modèle GR4J permet ainsi sur cet exemple de montrer que l'évolution observée sur les débits semble cohérente avec le comportement des pluies pour la région continentale Nord-Est, la transformation pluie-débit n'étant apparemment pas responsable de ce changement. Le second exemple d'application, avec le modèle MORDOR (pluie-neige-température) utilisé pour l'analyse des crues nivales des Alpes, montre un lien entre l'augmentation des températures et une modification de la saisonnalité des écoulements liés à la fonte (Renard, 2006).

\section{CONCLUSIONS ET PERSPECTIVES}

Le travail effectué dans le cadre de ce projet national a montré qu'aucune tendance climatique cohérente et généralisée à l'échelle de la France ne peut à ce jour être détectée sur l'évolution du régime des extrêmes hydrométriques. Le traitement d'un jeu d'environ 200 longues séries hydrométriques permet de s'affranchir des problèmes d'échantillonnage que l'on rencontre à partir d'une analyse sur un jeu réduit de données. Il faut insister sur plusieurs questions d'ordre méthodologique. Une attention particulière a été portée à 

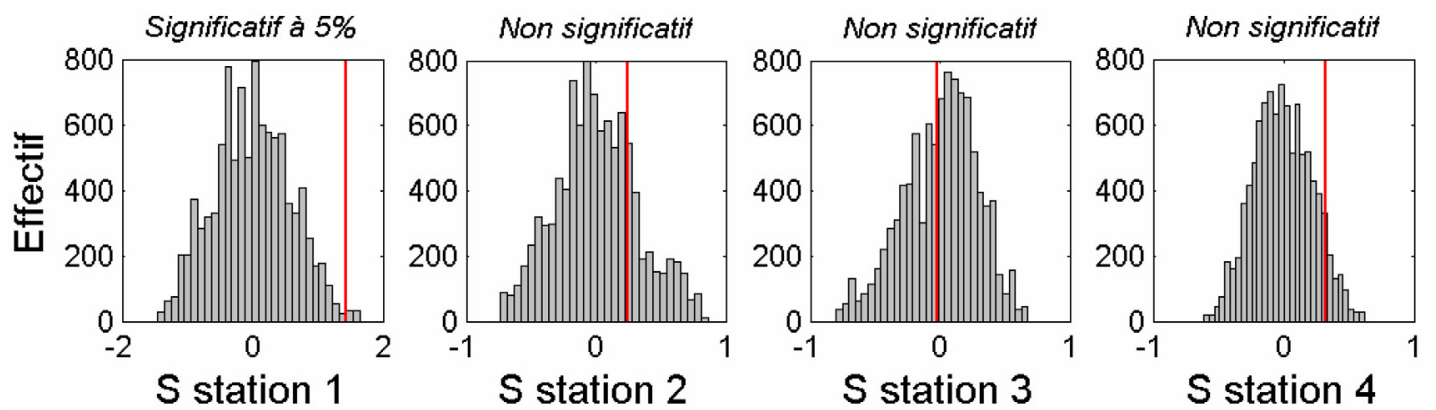

Figure 8 : Comparaison sur les quatre stations de la Moselle de la statistique $S$ observée avec la distribution simulée de cette statistique sous hypothèse de stationnarité de la relation pluie-débit.

la critique des données et a montré que près de la moitié des changements détectés dans une première analyse provenaient de biais métrologiques ou de la présence d'aménagements hydrauliques. Il est ainsi primordial de ne pas négliger cette analyse de critique des données pour la constitution d'un jeu de séries hydrométriques de référence. Un second point concerne les procédures de détection de changements, qui sont d'autant plus pertinentes qu'elles s'intéressent à la cohérence spatiale des évolutions. Une procédure semiparamétrique a été développée en ce sens, en prenant en compte également la redondance d'information liée à la dépendance spatiale. Elle repose sur une hypothèse de structure Gaussienne de la dépendance, qui été contrôlée sur le jeu de données analysé, mais qui gagnera certainement à être assouplie lors d'études ultérieures.

Des évolutions ont pu être détectées sur quelques régions hydro-climatiques et sont surtout à relier à l'augmentation des températures, avec pour conséquence une modification des crues nivales et du régime des étiages en zone de montagne. Pour les crues, seule la région Nord-Est présente une tendance à l'aggravation qui semble reliée à l'augmentation des précipitations. En conclusion, ces résultats ne prouvent évidemment pas l'absence d'impact du changement climatique, maintenant bien avéré sur les températures, mais démontrent la difficulté de discerner cette influence dans un signal à forte variabilité naturelle, avec d'autres sources de changement possible. Il convient de rester vigilant sur une évolution possible de l'aléa dans les prochaines années et de développer des outils de gestion du risque adaptés à un contexte non stationnaire. Renard et al. (2006a et b) ont proposé des premiers outils d'analyse probabiliste intégrant la non-stationnarité comme une incertitude supplémentaire. Des développements complémentaires sont envisagés en exploitant les travaux en cours de la communauté des statisticiens sur l'analyse multivariée des valeurs extrêmes.

\section{VI — REMERCIEMENTS}

Nous remercions les partenaires scientifiques impliqués dans le projet PNRH et le suivi de la thèse de B. Renard: P. Bois (LTHE/Grenoble), A. Dupeyrat, C. Laurent, J. Gailhard et E. Paquet (EDF LNHE/Chatou et DTG/Grenoble), O. Mestre (Météo-France ENM/Toulouse), H. Niel et L. Neppel
(HydroSciences/Montpellier), E. Sauquet (Cemagref/Lyon), C. Prudhomme (CEH Wallingford). Nous remercions également le MEDD pour la mise à disposition des données de la banque HYDRO, ainsi que les gestionnaires de stations hydrométriques contactés dans ce projet. Une pensée particulière pour Hélène Niel qui nous a quitté en décembre 2006.

\section{VII — BIBLIOGRAPHIE}

[1] Andreassian V., Parent E., Michel C. (2003) - A distribution-free test to detect gradual changes in watershed behavior. Water Resources Research. 39

[2] Champeaux J.L., Tamburini A. (1995) — Zonage climatique de la France à partir des séries de précipitations [1971-1990] du réseau climatologique d'État. La Météorologie. 14

[3] Douglas E.M., Vogel R.M., Krol C.N. (2000) - Trends in floods and low flows in the United States: impact of spatial correlation. Journal of Hydrology. 240 : 90-105

[4] Paquet E. (2004) - Evolution du modèle hydrologique MORDOR: modélisation du stock nival à différentes altitudes. La Houille Blanche. 75-82

[5] PERrin C. (2000) - Vers une amélioration d'un modèle global pluie-débit au travers d'une approche comparative. INP Grenoble, Cemagref Antony. $530 \mathrm{p}$

[6] Renard B. (2006) - Détection et prise en compte d'éventuels impacts du changement climatique sur les extrêmes hydrologiques en France. Thèse INP Grenoble, Cemagref Lyon, 20 sept. 361

[7] Renard B., Lang M., Bois P., Dupeyrat A., Mestre O., Niel H., Gailhard J., Laurent C., Neppel L., Sauquet E. (2006a) - Évolution des extrêmes hydrométriques en France à partir de séries observées. La Houille Blanche, $\mathrm{n}^{\circ} 6,48-54$

[8] Renard B., LANG M., Bois P. (2006) - Statistical analysis of extreme events in a non-stationary contact via a Bayesian framework : case study with peak-over-threshold data. Stochastic Environmental. Research and Risk Assessment. 21(2) : 97-112

[9] Renard B., Garreta V., Lang M. (2006) - An application of Bayesian analysis and Markov chain Monte Carlo methods to the estimation of a regional trend in annual maxima. Water Resour. Res. 42, W12422 : 17 p, doi :10.1029/2005WR004591

[10] Renard B., LANG M. (2007) - Use of a Gaussian copula for multivariate extreme value analysis : some case studies in hydrology. Advances in Water Resources. In press.

[11] Yue S., Wang C.Y. (2002) - Regional streamflow trend detection with consideration of both temporal and spatial correlation. International Journal of Climatology. 22 : 933-946 